A doublet microlens array for imaging micron-sized objects

This article has been downloaded from IOPscience. Please scroll down to see the full text article.

2011 J. Micromech. Microeng. 21105024

(http://iopscience.iop.org/0960-1317/21/10/105024)

View the table of contents for this issue, or go to the journal homepage for more

Download details:

IP Address: 141.211.173.82

The article was downloaded on 06/04/2012 at 16:04

Please note that terms and conditions apply. 


\title{
A doublet microlens array for imaging micron-sized objects
}

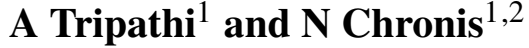 \\ ${ }^{1}$ Department of Mechanical Engineering, University of Michigan, Ann Arbor, MI, USA \\ ${ }^{2}$ Department of Biomedical Engineering, University of Michigan, Ann Arbor, MI, USA \\ E-mail: chronis@umich.edu
}

Received 20 May 2011, in final form 25 July 2011

Published 21 September 2011

Online at stacks.iop.org/JMM/21/105024

\begin{abstract}
We present a high-numerical aperture, doublet microlens array for imaging micron-sized objects. The proposed doublet architecture consists of glass microspheres trapped on a predefined array of silicon microholes and covered with a thin polymer layer. A standard silicon microfabrication process and a novel fluidic assembly technique were combined to obtain an array of $56 \mu \mathrm{m}$ diameter microlenses with a numerical aperture of $\sim 0.5$. Using such an array, we demonstrated brightfield and fluorescent image formation of objects directly on a CCD sensor without the use of intermediate lenses. The proposed technology is a significant advancement toward the unmet need of inexpensive, miniaturized optical modules which can be further integrated with lab-on-chip microfluidic devices and photonic chips for a variety of high-end imaging/detection applications.
\end{abstract}

(Some figures in this article are in colour only in the electronic version)

\section{Introduction}

Modern optical imaging-based research and industrial systems rely on the use of bulky and expensive objective lenses. Despite their superior performance in resolving submicron features under low-light conditions, these lenses can detect only one sample at a time (which lies within their field of view (FOV)), while manufacturing limitations do not allow miniaturization and integration with emerging micromachined devices, including miniature CCD sensors [1], photonic chips [2] and microfluidic biochips [3]. The development of onchip lens-based optical modules entails the miniaturization of these lenses while maintaining superb imaging quality. Such miniaturized, typically microfabricated lenses (also known as 'microlenses') can be used in displays [4-6], for optical coupling [7-9] and surface microstructuring [10] as well as in various biomedical imaging applications [11-13].

A number of microlens microfabrication approaches have been proposed over the past three decades. Photoresist reflow and transfer methods are the earliest methods for fabricating microlenses [14-16]. These microlenses have a low light collecting capability (indicated by a low numerical aperture (NA)) and are unable to form a resolved image of weakly light-emitting micron-sized objects. A modified approach called the boundary confined method has been proposed to obtain high-NA $(\mathrm{NA} \sim 0.54)$ microlenses [17]. Similar to other photoresist reflow approaches, this method requires accurate control of the surface tension of the photoresistsubstrate interface. Ink-jet printing of UV-curable polymers has been another approach for fabricating microlens arrays [18, 19]. This method requires an expensive setup for accurately dispensing a polymer on a rigid substrate. Recently, fabrication of high-NA (NA 0.37) microlens arrays using ink-jet printing of UV-curable polymers on hydrophobic glass substrates has been reported [20]. These processes require an elaborate control of the material properties (viscosity, surface tension) of the polymer as well as of the hydrophobicity of the substrate surface. Microfabrication of high-NA microlenses has also been proposed using focused ion beam milling and femtosecond laser direct writing technologies [21, 22]. These microfabrication processes are serial processes and require expensive equipments and experienced personnel. Soft lithography-based approaches in which optical materials are molded against rigid and elastomeric molds to obtain microlenses have also been demonstrated [23, 24]. These methods are simple to implement but the microlens quality is strongly dependent on the surface finish of the mold. Alternatively, planar, polymer-based arrays of microlenses can be microfabricated by UV-assisted curing of microfluidic networks [25]. These planar microlenses have a relatively high 
(A)

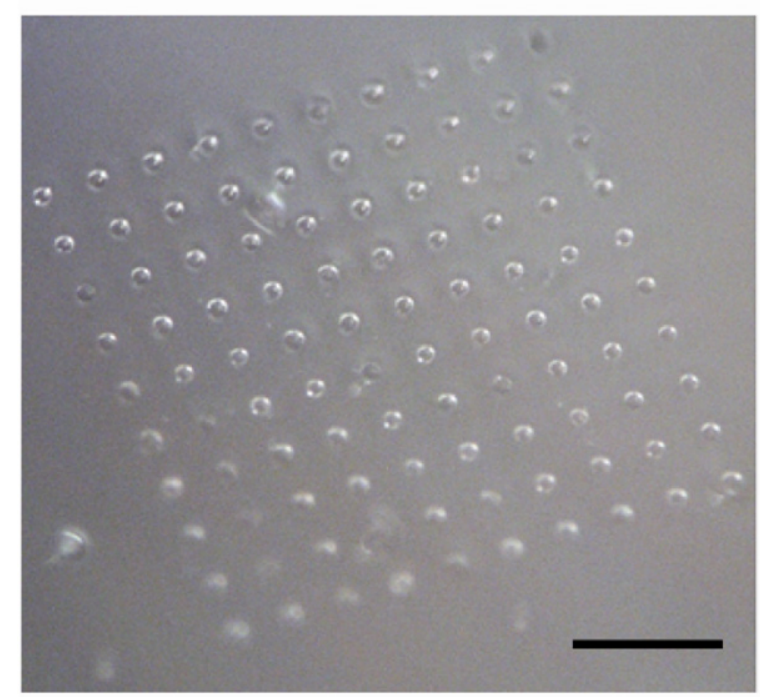

(B)

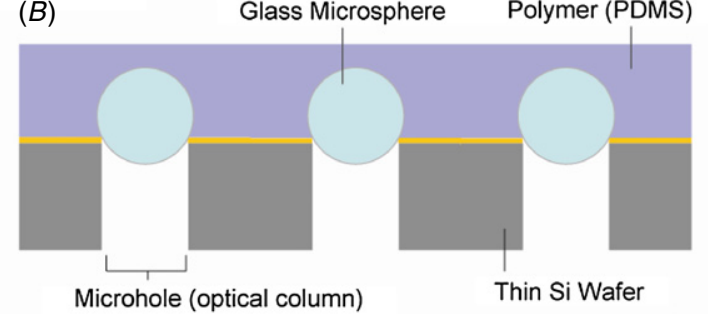

(C)

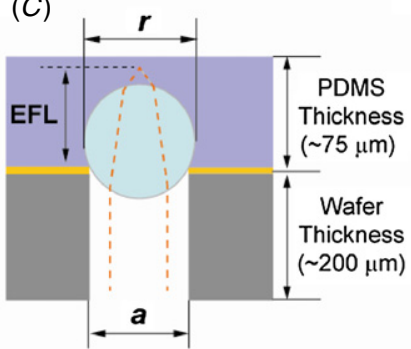

(D)

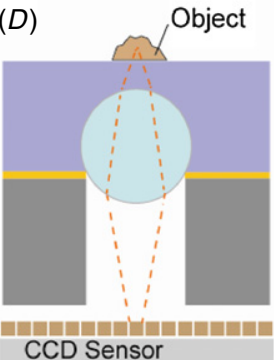

Figure 1. $(A)$ A $10 \times 10$ array of $56 \mu \mathrm{m}$ diameter doublet microlenses. Scale bar: $500 \mu \mathrm{m}$. $(B)$ A schematic of the cross section of the microlens array. The microlens diameter is defined by the diameter of the microhole. $(C)$ A collimated light beam is focused at a point (focal point) right below the top surface of the PDMS layer. The distance between the focal point and the silicon dioxide surface is the microlens effective focal length (EFL). $a$ is the microhole (microlens) diameter and $r$ is the microsphere diameter. $(D)$ Mechanism of image formation using the doublet microlens array. The object is placed on the surface of the microlens which forms a magnified image is formed directly on a CCD sensor.

NA (NA $\sim 0.3)$ when compared to other planar microlenses but are not amenable to an easy integration with other optical and microfluidic devices.

High-NA (NA 0.64) spherical microlenses have also been microfabricated by injecting SU-8 photoresist through microfabricated nozzles and utilizing surface tension to form micro-balls [26]. These microlenses largely vary in size due to fluctuations in the injection pressure and the contact angle between the photoresist and the substrate. Another process for spherical microlens fabrication involves polymer molding against isotropically etched silicon master molds [27]. The surface quality of the mold is highly dependent upon the precise balance of reagents used to etch the master mold. Finally, a number of high-NA, spherical microlens fabrication approaches employing the use of glass or polystyrene microspheres have been proposed [28, 29]. However, none of these microlenses have been shown to form the image on their own on an imaging sensor without the aid of additional optical elements. Several microlenses have been proposed for amplifying the light signal on the imaging sensor, hence increasing the effective NA of the imaging system [30-35]. However, all these approaches involve the use of macroscopic lenses in addition to the microlens arrays for image formation on the imaging sensor.

We propose a novel microfabrication approach for obtaining low-cost, high-NA arrays of doublet microlenses for imaging micron-sized objects without the need of any additional lenses. These microlenses are made out of glass microspheres with a transparent polymer spun on them. Planarizing one side of the microlenses with a transparent polymer layer enables an easy integration of the microlens array with lab-on-chip devices and other photonic chips. The microlens array is microfabricated using a combination of silicon micromachining, soft lithography and fluidic assembly. To our knowledge, this is the first demonstration of magnified image formation on a CCD sensor using a microlens array without use of intermediate lenses. An array of these doublet microlenses has the following advantages: (i) equivalent optical performance (resolution, NA) to a conventional microscope objective, (ii) simultaneous imaging of a large number of objects, (iii) easy integration with other micromachined modules, including microfluidic and optical chips, (iv) direct image formation on an imaging sensor without any extra lenses and (v) low microfabrication cost. With these advantages, we believe the proposed technology can be readily employed for applications involving imaging of micron-sized objects.

\section{Design and microfabrication of the doublet microlens array}

The proposed doublet microlens array consists of glass microspheres, fluidically assembled on top of an array of wafer-through microholes (figure 1). The microhole array captures/places the microspheres in a predefined pattern, while it creates a clear optical path for the collected light to reach the imaging sensor. The microlens array collects light originating from micron-sized objects and forms an image of these objects on the imaging sensor. It is intended to function as a magnifying lens: objects placed at a plane slightly below the focal plane of the microlens array appear magnified at the imaging sensor. To ensure that the magnified image is in focus on the plane of the sensor the sensor's spatial position has to be adjusted in the vertical direction using a translational manipulator. 


\section{PECVD Silicon Dioxide Deposition}

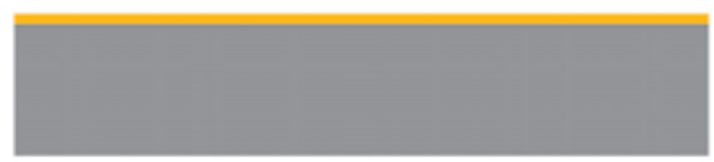

2. Microhole Patterning and DRIE
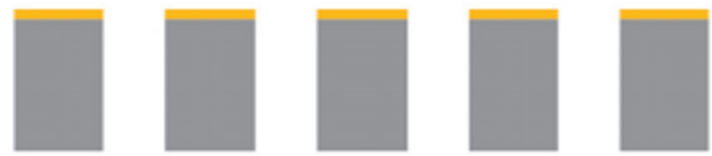

\section{Lapping and CMP}

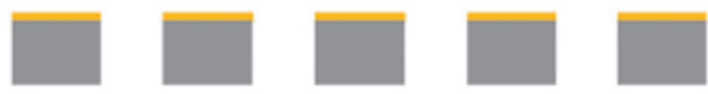

\section{Fluidic Assembly of Microspheres}

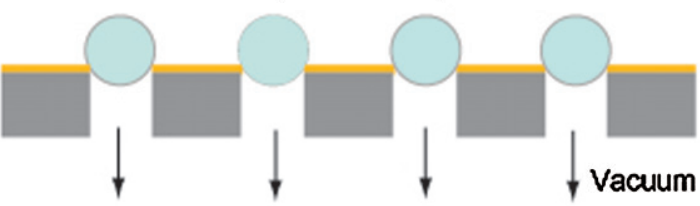

\section{PDMS Spinning and Curing}

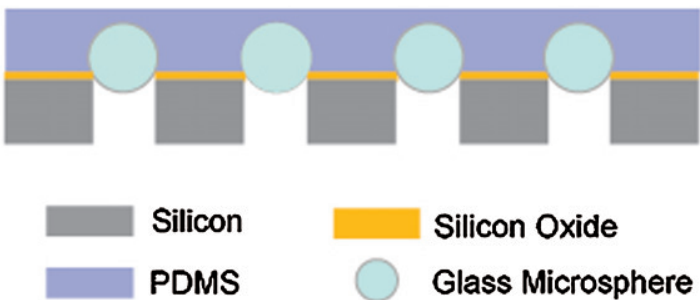

Figure 2. Microfabrication process of the doublet microlens array. Key concept is the fluidic assembly of glass microspheres (step 4).

The microfabrication of the doublet microlens array involves three steps (figure 2): (i) microfabrication of the microhole array, (ii) assembly of glass microspheres on the array and (iii) spinning of a polymer layer on the captured microspheres to form the doublet microlenses. Specifically, a $2 \mu \mathrm{m}$ thick PECVD silicon dioxide layer is initially deposited on an $\sim 500 \mu \mathrm{m}$ thick silicon substrate. An array of 56 $\mu \mathrm{m}$ diameter microholes is then patterned and etched on the oxide layer using standard photolithography and reactive ion etching (RIE). Deep reactive ion etching (DRIE) is further employed to fabricate wafer-through circular microholes in the silicon substrate. Subsequently, the silicon substrate is thinned down to $200 \mu \mathrm{m}$ using a combination of lapping and chemical-mechanical polishing (CMP). An aqueous solution containing glass microspheres $(60 \mu \mathrm{m}$ nominal diameter, refractive index 1.51 at a wavelength of $589 \mathrm{~nm}$; catalog no 02718-AB, Structure Probe, Inc.) is then dispensed on the oxide-coated silicon surface. A suction force is subsequently applied from the other side of the substrate to assemble and trap the glass microspheres atop of the microhole array. Doublet microlenses are finally obtained by spinning and curing $\left(65^{\circ} \mathrm{C}, 1 \mathrm{~h}\right.$ on a hot plate $)$ an $\sim 75 \mu \mathrm{m}$ thick (spun at $1600 \mathrm{rpm}$ ) layer of polydimethylsiloxane (PDMS) elastomer on top of the array.

\section{Results and discussion}

We performed optical simulations using OSLO software to ascertain the effective focal length (EFL) of the fabricated microlenses (for all simulations, we assumed a wavelength of $580 \mathrm{~nm}$ ). We defined the microlens EFL as the distance between the best focused plane of a collimated light beam passing through a doublet microlens and the silicon dioxidecoated wafer surface (figure $1(C)$ ). A refractive index of 1.41 and 1.51 was used for the PDMS and the glass microspheres, respectively. EFL values of 65-75 $\mu \mathrm{m}$ were obtained for glass microspheres ranging 57-63 $\mu \mathrm{m}$ in diameter. Experimental EFL values were obtained for individual microlenses in the array using collimated white light (see [25] for details on the experimental setup used to measure the EFL). The EFL measurement process involved obtaining the best focused plane by capturing a stack of images along the microlens axis and finding the plane with the maximum light intensity. A $40 \times(0.6 \mathrm{NA})$ microscope objective was used to image the best focused plane. EFL values measured for 20 microlenses in the microlens array varied from 63 to $71 \mu \mathrm{m}$. These values correlated strongly with the EFL range obtained from optical simulations. Such a variation on the measured EFL between microlenses can be attributed to variations of the microsphere diameter (5\%, given by the manufacturer). We also performed optical simulations to assess the dependence of the EFL on the PDMS curing conditions (temperature, time) as those are known to affect the PDMS refractive index [36]. For two extreme curing cases $\left(48 \mathrm{~h}\right.$ at $25{ }^{\circ} \mathrm{C}$, and $1 \mathrm{~h}$ at $150{ }^{\circ} \mathrm{C}$ ), the EFL was found to change by less than $1 \mu \mathrm{m}$ (assuming a change in the PDMS refractive index from 1.416 to 1.472 [36]). These results show that the EFL of our microlenses is not significantly affected by the PDMS curing conditions.

Furthermore, we used optical simulations to estimate the NA of the doublet microlenses as a function of microhole diameter $(a)$ to microsphere diameter $(r)$ ratio (figure 3 ). For our fabricated microlenses $(a=56 \mu \mathrm{m}, r=60 \mu \mathrm{m}, a / r=$ $0.93)$, a NA of 0.495 was obtained. This NA value is very close to the maximum theoretical NA value $(0.53, a / r=$ 1.0) achievable using this microfabrication method. It should be emphasized that objectives with a NA of 0.350.4 are commonly used in microscopy for imaging cells and tissues [37].

To measure the depth of focus (DOF) of the doublet microlenses, we obtained the axial light intensity profile of a collimated light beam that was focused through the microlens array (figure 4). The collimated light beam was generated by placing a point source of light (a halogen lamp) $25-30 \mathrm{~cm}$ away from the microlens array. The average light intensity was obtained for imaging planes above and below the best focused plane (the focal plane) and normalized with respect to the average light intensity at the best focused plane (that corresponded to the maximum average intensity). Intensity 


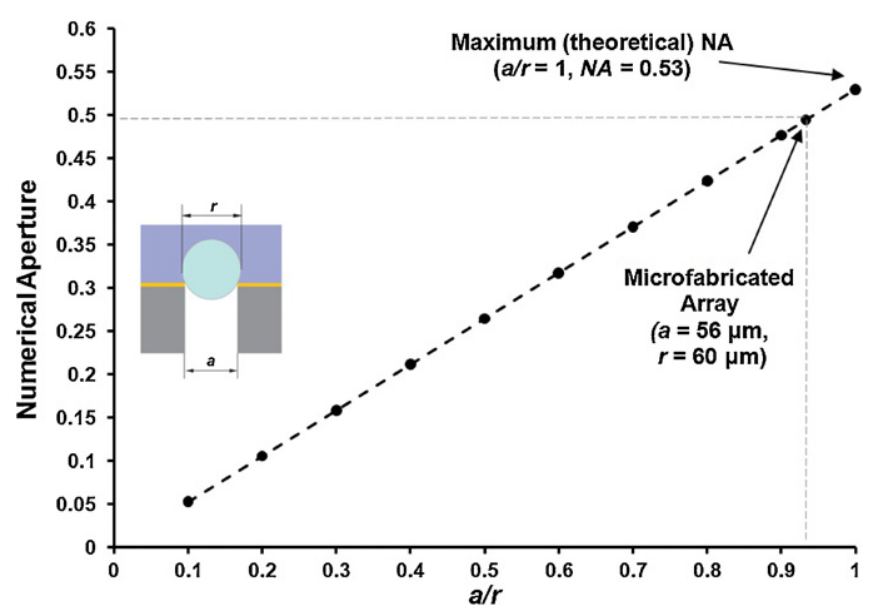

Figure 3. Simulation results depicting the dependence of the NA on the microhole to microsphere diameter ratio $(a / r)$. A NA value of 0.495 was estimated for our microfabricated microlenses.

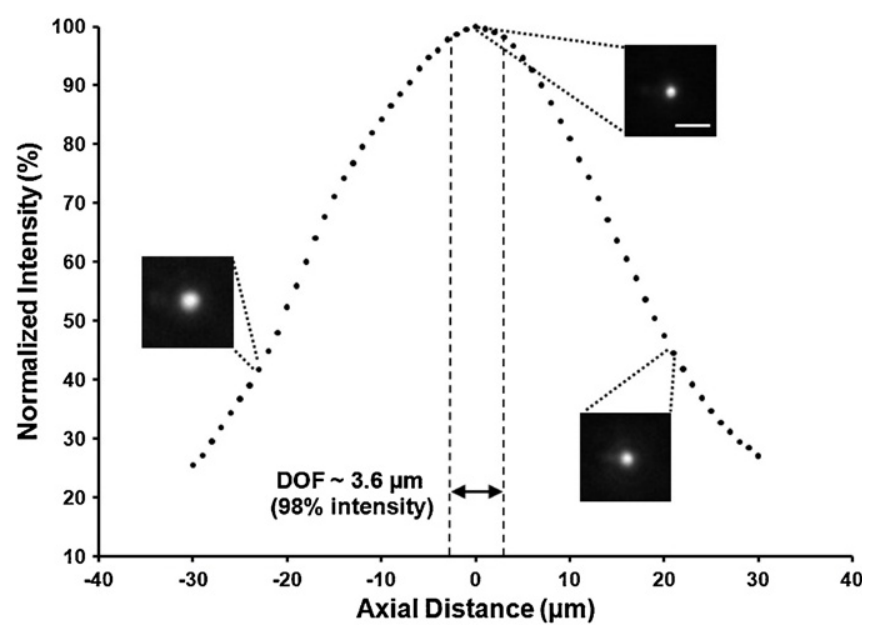

Figure 4. Normalized intensity (with respect to the

background-corrected average intensity at the best focused plane) of a doublet microlens as a function of the axial distance from the best focused plane. The images depict a focused light beam at different axial planes. Scale bar: $10 \mu \mathrm{m}$.

values for all imaging planes were background-corrected. The DOF of the fabricated doublet microlenses $(a / r=0.93$, $\mathrm{NA} \sim 0.495$ ) was estimated to be $\sim 3.6 \mu \mathrm{m}$ [38]. Within this distance, the average light intensity decreased by $2 \%$ from its maximum value (at the best focused plane).

Low $(4 \times)$ and high $(40 \times)$ magnification measurements were taken from a $4 \times 4$ microlens array and from a single microlens respectively to obtain the point spread function (PSF) (figure 5). The $4 \times 4$ array had 16 spatially distinguishable intensity peaks (figure $5(A)$ ), observed at the center of each microlens. The average value of the normalized intensity from those 16 peaks was $82.3 \%$ (standard deviation $19.6 \%$ ), ranging from $44 \%$ to $100 \%$ between individual microlenses. This non-uniformity in the peak values can be attributed to the EFL variation discussed earlier in this section.

As described above, a major advantage of the proposed microlens array is its capability to form an image directly on an imaging sensor without intermediate lenses. To demonstrate
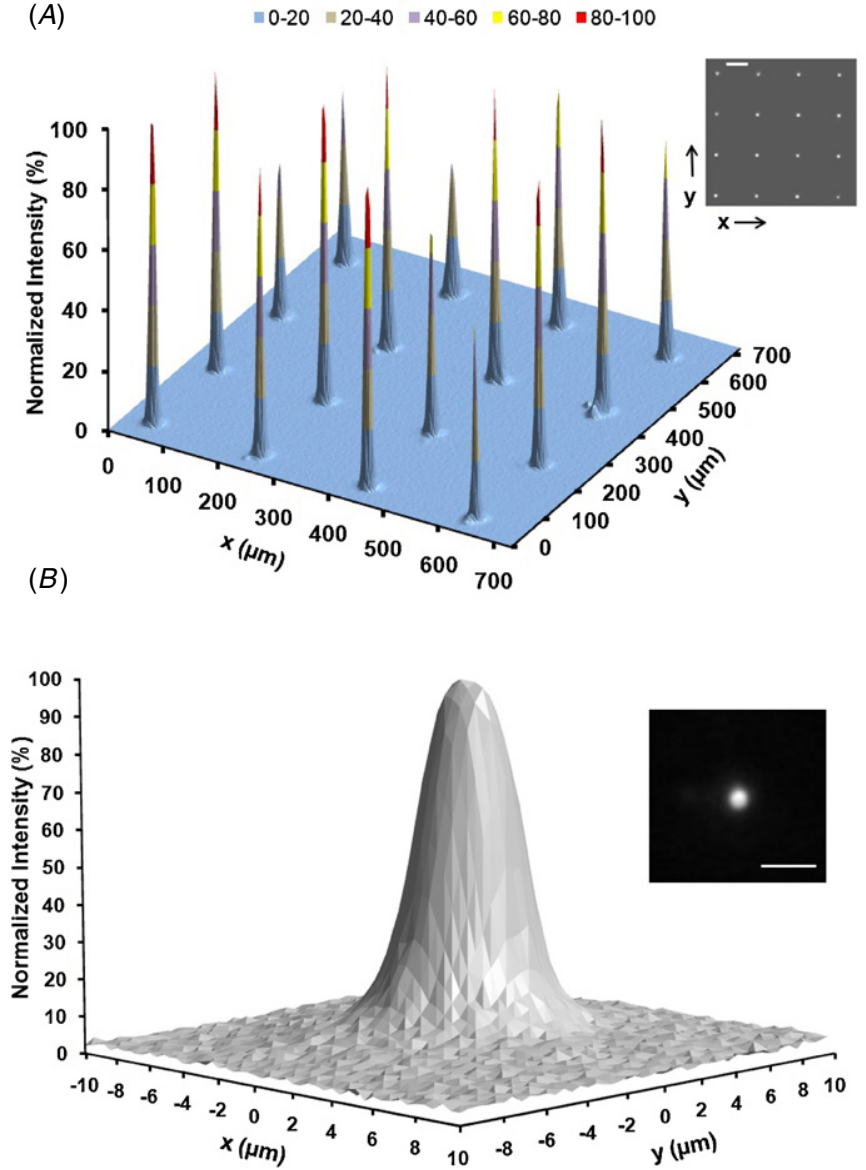

Figure 5. (A) PSF of a $4 \times 4$ microlens array. Scale bar of the image: $100 \mu \mathrm{m}$. (B) PSF of an individual microlens. Scale bar of the image: $10 \mu \mathrm{m}$. $(A)$ and $(B)$ plots were obtained using a $4 \times$ $(\mathrm{NA}=0.15)$ and a $40 \times(\mathrm{NA}=0.6)$ microscope objective, respectively. In both $(A)$ and $(B)$, normalized intensity values at a particular $x y$ point were obtained by normalizing the backgroundcorrected light intensity at that point with the maximum value in the entire image.

the image formation capability and magnification achieved using these microlenses, we used a microlens array to image resolution patterns etched on a chrome layer, deposited on a glass substrate (figure 6). A $640 \times 480$ CCD sensor chip (5.6 $\mu \mathrm{m}$ pixel size), dismantled from a web camera (Logitech QuickCam 3000), and a custom experimental setup utilizing $x$-, $y$ - and $z$-axis manipulators were used to acquire images and to align the patterns-microlens assembly with the imaging sensor chip (figure 6(A)). A white light source was used to illuminate the resolution patterns. In this brightfield, transmission imaging mode, the microlens array was able to resolve various 1 and $2 \mu \mathrm{m}$ resolution patterns (figure $6(B)(\mathrm{I})$ (III)). The theoretical resolution was calculated to be $0.45-$ $0.71 \mu \mathrm{m}$ (for a 450-700 nm wavelength) [38].

We also demonstrated the use of these microlenses for fluorescent imaging of micron-sized objects. We obtained a magnified, fluorescent image of a $4 \mu \mathrm{m}$ diameter polystyrene bead (excitation peak $505 \mathrm{~nm}$, emission peak $515 \mathrm{~nm}$, catalog no F-8859, Invitrogen, Inc.) using a slightly modified setup for fluorescence imaging (figure $6(B)(I V)$ ). The fluorescent beads were illuminated with blue light obtained after passing 


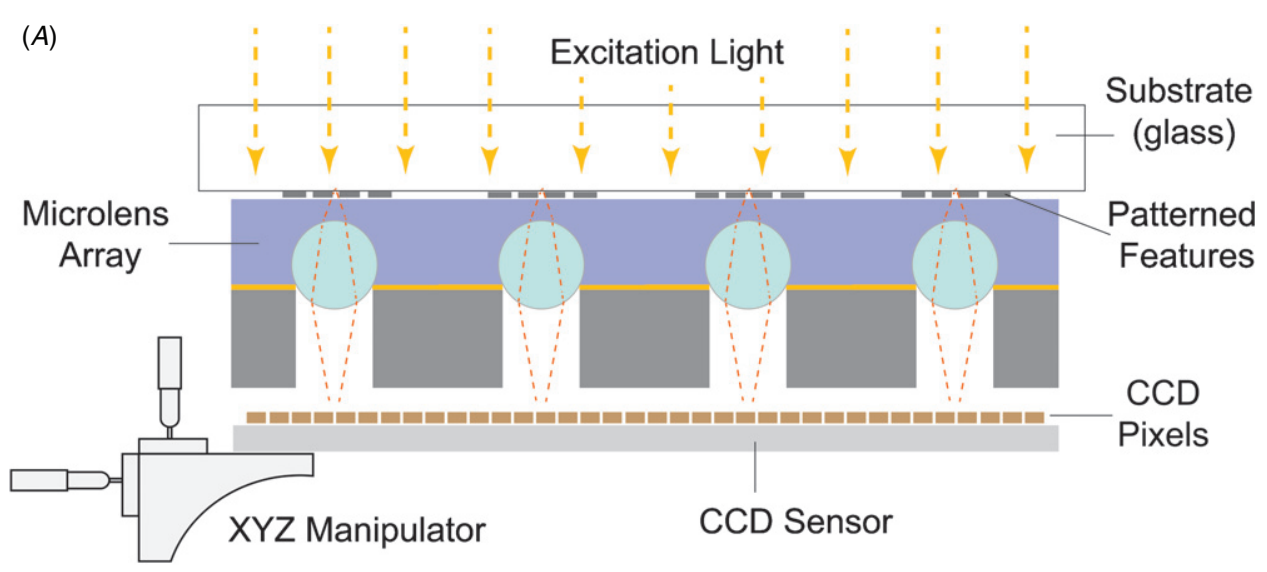

(B)
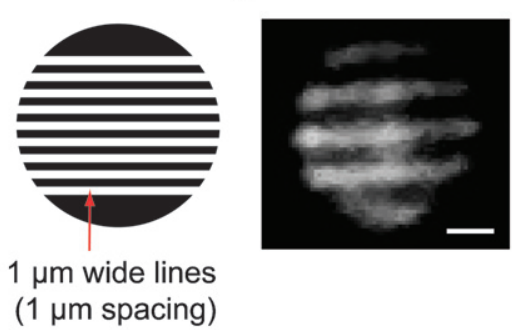

(III)

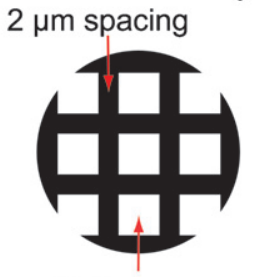

$5 \times 5 \mu \mathrm{m}$ square

(I)
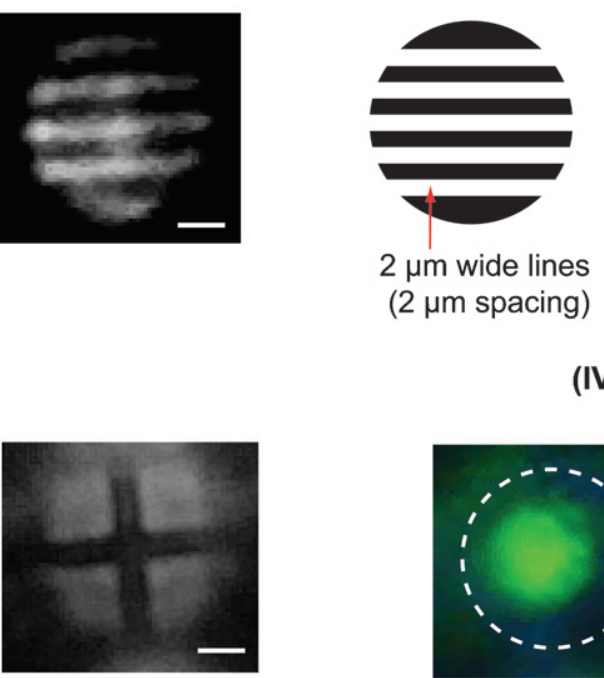

(IV)

(II)

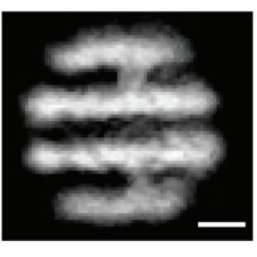

(2 $\mu \mathrm{m}$ spacing)

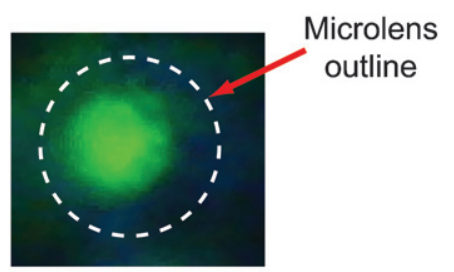

Figure 6. (A) Schematic of the setup for imaging micron-sized resolution patterns using the doublet microlens array. (B) (I), (II) Brightfield, transmission images of 1 and $2 \mu \mathrm{m}$ line resolution patterns respectively using a $56 \mu \mathrm{m}$ diameter microlens (NA $\sim 0.495$ ).

Equally spaced, $1 \mu \mathrm{m}$ wide lines are clearly resolved by the doublet microlens. Scale bar: $20 \mu \mathrm{m}$. (III) Brightfield, transmission image of a $5 \mu \mathrm{m}$ square grid. Scale bar: $20 \mu \mathrm{m}$. (IV) Fluorescence image of a polystyrene bead (4 $\mu \mathrm{m}$ in diameter).

white light (originated by a halogen lamp) through an optical band-pass filter (XF1073 475AF40; Omega Optical). A thin $(\sim 100 \mu \mathrm{m})$ long-pass emission filter (Yellow 12 Kodak Wratten color filter) was inserted between the microlens array and the CCD sensor to block the excitation light. The fluorescent image obtained for the bead was magnified by a factor of $\sim 6$. At this $6 \times$ magnification, a $56 \mu \mathrm{m}$ diameter microlens (with a $60 \mu \mathrm{m}$ microsphere) is estimated to have a FOV of $\sim 9.3 \mu \mathrm{m}$ (FOV $=$ microhole diameter $/$ magnification $)$. The dimensions of the objects used for brightfield and fluorescence imaging correspond to an average cell diameter (e.g. blood cell) and hence the above imaging results validate the potential use of these microlenses for imaging biological micron-sized objects (cells, tissues, etc) directly on a CCD sensor.

\section{Conclusions}

We have proposed a novel doublet microlens array microfabrication approach for the direct visualization of micron-sized objects. Using these microlenses we have demonstrated direct image formation on a CCD sensor without additional optical elements. These microlenses resolved $1 \mu \mathrm{m}$ resolution patterns and had a NA of $\sim 0.495$. As a result, they can be considered as miniaturized microscope objectives and they provide a cheaper alternative for bulky and expensive microscope optics. They can readily find biomedical applications in imaging of cellular and subcellular components. Integration of this micro-optical module with various photonic and microfluidic devices will pave the way for the development of next generation of integrated biomedical imaging devices.

\section{Acknowledgments}

This work was supported by the National Institutes of Health (NIH) Director's New Innovator Award \#DP2OD006458. All the devices were fabricated at the Lurie Nanofabrication Facility at the University of Michigan. We also thank Philip 
Choi, Trushal Vijaykumar Chokshi, Mostafa Ghannad-Rezaie and Eleni Gourgou for useful discussions.

\section{References}

[1] Itakura K, Nobusada T, Kokusenya N, Nagayoshi R and Ozaki R 2000 A 1-mm 50 k-Pixel IT CCD image sensor for miniature camera system IEEE Trans. Electron Devices 4765

[2] Yang W, Conkey D B, Wu B, Yin D, Hawkins A R and Schmidt H 2007 Atomic spectroscopy on a chip Nature Photonics 1331

[3] Verpoorte E 2003 Chip vision-optics for microchips Lab Chip $342 \mathrm{~N}-52 \mathrm{~N}$

[4] Shieh H P D, Huang Y P and Chien K W 2005 Micro-optics for liquid crystal displays applications J. Disp. Technol. 162

[5] Peng H, Ho Y L, Yu X J, Wong M and Kwok H S 2005 Coupling efficiency enhancement in organic light-emitting devices using microlens array - theory and experiment J. Disp. Technol. 1278

[6] Lim J et al 2006 Enhanced out-coupling factor of microcavity organic light-emitting devices with irregular microlens array Opt. Express $\mathbf{1 4} 6564$

[7] Shen S C, Pan C T, Chou H P and Chou M C 2001 Batch assembly micro-ball lens array for Si-based optical coupling platform in free space Opt. Rev. 8373

[8] He M, Yuan X C, Ngo N, Bu J and Tao S 2003 Low-cost and efficient coupling technique using reflowed sol-gel microlens Opt. Express 111621

[9] Shen S C, Pan C T, Liu K H, Chao C H and Huang J C 2009 Fabrication of an eyeball-like spherical micro-lens array using extrusion for optical fiber coupling J. Micromech. Microeng. 19125017

[10] O'Connell C, Sherlock R and Glynn T J 2010 Fabrication of a reusable microlens array for laser-based structuring $O p t$. Eng. 49014201

[11] Aljasem K, Werber A, Seifert A and Zappe H 2008 Fiber optic tunable probe for endoscopic optical coherence tomography J. Opt. A: Pure Appl. Opt. 10044012

[12] Barretto R P J, Messerschmidt B and Schnitzer M J 2009 In vivo fluorescence imaging with high-resolution microlenses Nature Methods 6511

[13] Mogi T, Hatakeyama K, Taguchi T, Wake H, Tanaami T, Hosokawa M, Tanaka T and Matsunaga T 2011 Real-time detection of DNA hybridization on microarray using a CCD-based imaging system equipped with a rotated microlens array disk Biosens. Bioelectron. 261942

[14] Popovic Z D, Sprague R A and Connell G A N 1988 Technique for monolithic fabrication of microlens arrays Appl. Opt. 271281

[15] Fletcher D A, Crozier K B, Guarini K W, Minne S C, Kino G S, Quate C F and Goodson K E 2001 Microfabricated silicon solid immersion lens J. Microelectromech. Syst. 10450

[16] Wu M H and Whitesides G M 2002 Fabrication of twodimensional arrays of microlenses and their applications in photolithography J. Micromech. Microeng. 12747

[17] Hsieh H T and Su G D J 2010 A novel boundary-confined method for high numerical aperture microlens array fabrication J. Micromech. Microeng. 20035023

[18] Biehl S, Danzebrink R, Oliveira P and Aegerter M A 1998 Refractive microlens fabrication by ink-jet process J. Sol-Gel Sci. Technol. 13 177-82

[19] Tien C H, Hung C H and Yu T H 2009 Microlens arrays by direct-writing inkjet print for LCD backlighting applications J. Disp. Technol. 5 147-51
[20] Kim J Y, Brauer N B, Fakhfouri V, Boiko D L, Charbon E, Grutzner G and Brugger J 2011 Hybrid polymer microlens arrays with high numerical apertures fabricated using simple ink-jet printing technique Opt. Mater. Express 1259

[21] Chiu Y, Huang C H and Hsu Y C 2007 High numerical-aperture microlens fabricated by focused ion beam milling Proc. SPIE 6620 6620L

[22] Wu D, Wu S Z, Niu L G, Chen Q D, Wang R, Song J F, Fang H H and Sun H B 2010 High numerical aperture microlens arrays of close packing Appl. Phys. Lett. 97031109

[23] Kunnavakkam M V, Houlihan F M, Schlax M, Liddle J A, Kolodner P, Nalamasu O and Rogers J A 2003 Low-cost, low-loss microlens arrays fabricated by soft-lithography replication process Appl. Phys. Lett. 821152

[24] Yuan X C, Yu W X, He M, Bu J, Cheong W C, Niu H B and Peng X 2005 Soft-lithography-enabled fabrication of large numerical aperture refractive microlens array in hybrid $\mathrm{SiO}_{2}-\mathrm{TiO}_{2}$ sol-gel glass Appl. Phys. Lett. 86114102

[25] Tripathi A, Chokshi T V and Chronis N 2009 A high numerical aperture, polymer-based, planar microlens array Opt. Express 1719908

[26] Huang Y R and Chou H P 2004 Fabrication of ball-type spherical microlens array for optical fiber coupling Proc. SPIE 5347 281-6

[27] Albero J, Nieradko L, Gorecki C, Ottevaere H, Gomez V, Thienpont H, Pietarinen J, Päivänranta B and Passilly N 2009 Fabrication of spherical microlenses by a combination of isotropic wet etching of silicon and molding techniques Opt. Express 176283

[28] Wu M H and Whitesides G M 2001 Fabrication of arrays of two-dimensional micropatterns using microspheres as lenses for projection photolithography Appl. Phys. Lett. 782273

[29] Huang J Y, Lu Y S and Yeh J A 2006 Self-assembled high NA microlens arrays using global dielectricphoretic energy wells Opt. Express 1410779

[30] Schlingloff G, Kiel H J and Schober A 1998 Microlenses as amplification for CCD-based detection devices for screening applications in biology, biochemistry, and chemistry Appl. Opt. 371930

[31] Ren H, Fan Y H and Wu S T 2004 Liquid-crystal microlens arrays using patterned polymer networks Opt. Lett. 291608

[32] Chen C Y, Su W C, Wang Y F and Chen C H 2010 Reduction of distortion aberration in imaging systems by using a microlens array Opt. Commun. 2832798

[33] Guo R, Yuan D and Das S 2011 Large-area microlens arrays fabricated on flexible polycarbonate sheets via single-step laser interference ablation J. Micromech. Microeng. 21015010

[34] Malinauskas M A, Gilbergs H, Zukauskas A, Purlys V, Paipulas D and Gadonas R 2010 A femtosecond laser-induced two-photon photopolymerization technique for structuring microlenses J. Opt. 12035204

[35] Schonbrun E, Steinvurzel P E and Crozier K B 2011 A microfluidic fluorescence measurement system using an astigmatic diffractive microlens array Opt. Express 191385

[36] Chang-Yen D A, Eich R K and Gale B K 2005 A monolithic PDMS waveguide system fabricated using soft-lithography techniques J. Lightwave Technol. 232088

[37] Neumann B, Held M, Liebel U, Erfle H, Rogers P, Pepperkok R and Ellenberg J 2006 High-throughput RNAi screening by time-lapse imaging of live human cells Nature Methods 3385

[38] Inoué S 1997 Video Microscopy: the Fundamentals (New York: Plenum) 